\title{
Multi-color photometry in wide field of the Galactic globular cluster NGC $3201^{\star, \star \star}$
}

\author{
V. Kravtsov ${ }^{1,2}$, G. Alcaíno ${ }^{3}$, G. Marconi ${ }^{4}$, and F. Alvarado ${ }^{3}$ \\ 1 Instituto de Astronomía, Universidad Católica del Norte, Avenida Angamos 0610, Antofagasta, Chile \\ e-mail: vkravtsov@ucn.cl \\ 2 Sternberg Astronomical Institute, University Avenue 13, 119899 Moscow, Russia \\ 3 Isaac Newton Institute of Chile, Ministerio de Educación de Chile, Casilla 8-9, Correo 9, Santiago, Chile \\ e-mail: inewton@terra.cl, falvarad@eso.org \\ 4 ESO - European Southern Observatory, Alonso de Cordova 3107, Vitacura, Santiago, Chile \\ e-mail: gmarconi@eso.org
}

Received 9 July 2008 / Accepted 24 December 2008

\section{ABSTRACT}

\begin{abstract}
Aims. This paper aims at further studying one of the nearby Galactic globular clusters (GCs), NGC 3201. It is known to experience notable irregular variability of reddening across its face. By relying on our previous studies and findings and by developing them, we focus on the brighter sequences of the color-magnitude diagram (CMD) and on the cluster's characteristics.

Methods. We carried out and analyzed new multi-color photometry of NGC 3201 in UBVI reaching below the turnoff point in all passbands in a fairly large cluster field, about $14^{\prime} \times 14^{\prime}$. To achieve more reliable results and conclusions, we reduced the negative impact of the irregularly varying reddening and contamination by field stars.

Results. With this aim we first estimated mean reddening in different zones of the studied cluster field and then took its variations into account, by reducing them to the same level. We estimated metallicity of NGC 3201 using a new metallicity indicator related to $U$-based CMDs, recently proposed by us. We find $[\mathrm{Fe} / \mathrm{H}]_{Z W}=-1.54 \pm 0.12$ dex, which falls between extreme estimates of the cluster's metallicity obtained using different methods or indicators. Also, the location of the RGB bump on the branch corresponds to $[\mathrm{Fe} / \mathrm{H}]_{Z W}=-1.46 \pm 0.15$ dex. We isolate 73 probable blue straggler (BS) candidates, the largest population found in NGC 3201 so far. They are more centrally concentrated than the lower red giants at the $99.2 \%$ level. Their position in the two-color diagram assumes that presumably none of them belongs to BSa of collisional origin. The luminosity function (LF) of the RGB and its features in the low part of the branch are examined and discussed. We also resolve some of the contradictory results of previous publications.
\end{abstract}

Key words. globular clusters: general - globular clusters: individual: NGC 3201

\section{Introduction}

In the system of Galactic globular clusters (GCs), the southern cluster NGC $3201\left(\alpha_{2000}=10^{\mathrm{h}} 17^{\mathrm{m}} 36.8\right.$ and $\delta_{2000}=$ $\left.-46^{\circ} 24^{\prime} 40^{\prime \prime}\right)$ is known to have the highest radial velocity, $494.0 \mathrm{~km} \mathrm{~s}^{-1}$ (Harris 1996), and a retrograde Galactic orbit. It is also one of the nearby clusters. However, it can hardly be referred to as the best and frequently studied GCs. Irregular and highly variable reddening observed in the field of the cluster (Schlegel et al. 1998) seems to be the major factor responsible for such an outcome, since it affects the reliability of results. According to the extinction map obtained by von Braun \& Mateo (2001) in a wide cluster field, the reddening shows not only large-scale variations, up to $\sim 0.2 \mathrm{mag}$ in the $V-I$ across the cluster face, but it also can notably fluctuate even on a small scale, of 1 arc-minute or so. As a consequence, unfortunately, this very probably leads to some residual spread of stars even in differentially dereddened color-magnitude diagrams (CMDs). Being based on different methods of accounting for differential

\footnotetext{
* Based on observations with the $1.3 \mathrm{~m}$ Warsaw telescope at Las Campanas Observatory.

$\star \star$ Table of photometric measurements is only available in electronic form at CDS via anonymous ftp to

cdsarc.u-strasbg.fr $(130.79 .128 .5)$ or via

http://cdsweb.u-strasbg.fr/cgi-bin/qcat?J/A+A/497/371
}

reddening, such CMDs of NGC 3201, were obtained by von Braun \& Mateo (2001) and Layden \& Sarajedini (2003).

Here, we omit a separate and detailed overview of the publications devoted to NGC 3201 and of the respective results obtained to date. We refer to the majority of them elsewhere in our paper in the context of the problems we study; however, the most progress appears to have been achieved in investigations of the cluster's variables, as compared to other issues, thanks to a series of dedicated publications at the beginning of the 2000s (von Braun \& Mateo 2002; Piersimoni et al. 2002; Mazur et al. 2003; Layden \& Sarajedini 2003).

After a number of previous works on NGC 3201, made by some of us in different co-authorship, we address NGC 3201 again, by carrying out and analyzing new multi-color photometry in a wide cluster field, including measurements in the $U$ bandpass. Our present study deals with several issues but emphasizes (1) estimates of cluster metallicity; (2) probable population of blue stragglers (BSs); and (3) evolution of red giant branch (RGB) stars. We note again that doing photometry in GCs in the $U$ passband is relatively rare among current investigations of Galactic GCs. Hence, the photometric properties of stellar populations of different evolutionary sequences and in metallicity range typical for GCs remain less thoroughly studied in the $U$ passband and $U$-based colors. 

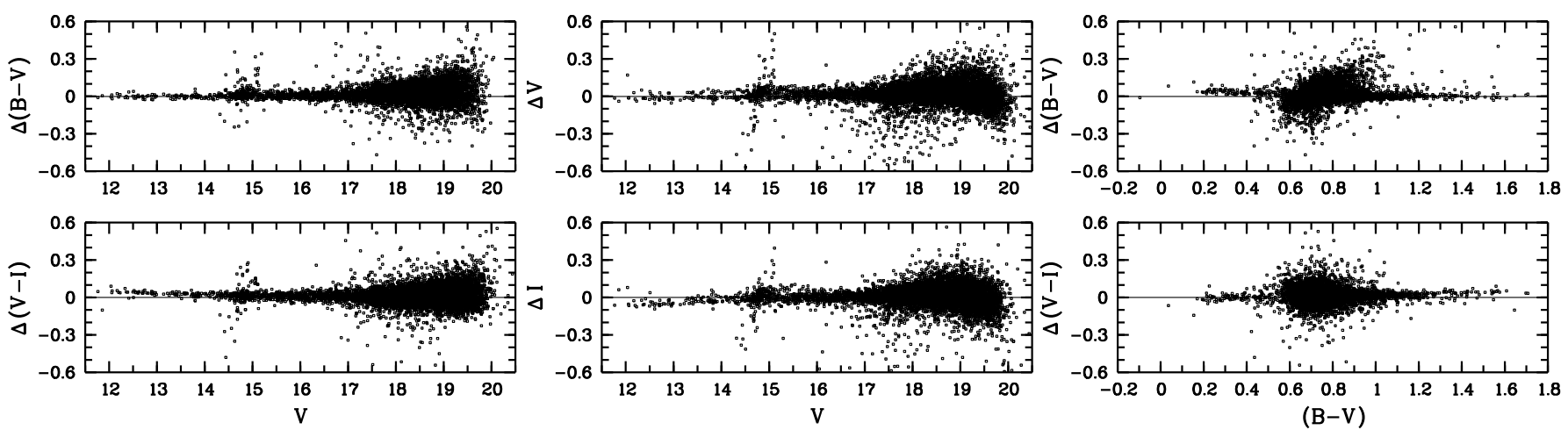

Fig. 1. Comparison of our present photometry of NGC 3201 and photometry by Layden \& Sarajedini (2003) of the cluster. The differences in colors and magnitudes are in the order of our data minus those of Layden \& Sarajedini.

\section{Observations and data reduction}

The observations were acquired on three nights, December 30/31 1997 and January 01/02, 02/03 1998, with the $1.3 \mathrm{~m}$ Warsaw telescope, Las Campanas Observatory, using a set of $U B V I$ filters and a $2048 \times 2048$ CCD camera with a gain $=$ 3.8 and a readout noise of $5.5 e^{-}$rms. The array scale was $0{ }^{\prime} 417$ pixel $^{-1}$, giving a field of view of $14^{\prime} \times 14^{\prime}$. The center of the measured field of NGC 3201 was approximately $20^{\prime \prime}$ to the east and 59" to the north of the cluster center. Flat-field, bias, and dark frames were taken twice per night at the beginning and the end of each one. We took a total of 6 frames in $U$ (exposure time from $300 \mathrm{~s}$ to $900 \mathrm{~s}), 7$ frames in $B(20 \mathrm{~s}$ to $180 \mathrm{~s}), 7$ frames in $V$ $(20 \mathrm{~s}$ to $120 \mathrm{~s})$, and 7 frames in $I(20 \mathrm{~s}$ to $120 \mathrm{~s})$. All exposures were taken at air mass varying insignificantly between 1.048 and 1.061. The average seeing estimated from the observations was about 1 .'0-1.'2.

The reductions of CCD photometry were performed at the Isaac Newton Institute and at the European Southern Observatory, ESO, Santiago, Chile. The stellar photometry was carried out separately for all frames using DAOPHOT/ALLSTAR (Stetson 1987, 1991), and the IRAF package ${ }^{1}$. The program stars were detected and measured by applying the usual procedures. To obtain the PSF, 20 to 30 stars in each frame, bright but far from saturation, were selected among those having no neighbors or defects within the PSF radius. We find that, among standard PSFs provided by DAOPHOT, the PENNY2 function enables us to handle the aberrations specific to individual frames most effectively. The instrumental magnitudes and colors $(v, u-b, b-v$, $v-i$ ) obtained for the measured stars were then transformed to the standards system $(V, U-B, B-V, V-I)$. Following the standard approach used at the Isaac Newton Institute within the framework of photometric CCD studies of GCs, we relied on photoelectric standard stars set in the cluster field. A total of 17 standard stars were used, of which 10 stars $(U B V I)$ are from Alcaíno \& Liller (1984) with new values for four of them, published by Alcaíno et al. $(1989)^{2}$, plus seven stars in the cluster field from Lee (1977) ${ }^{3}$.

We use the least squares method to calculate a straight line that best fits the data for these standards stars. The formulae used in this study to bring instrumental magnitudes and colors to the

\footnotetext{
${ }^{1}$ IRAF is distributed by the National Optical Astronomy Observatory, which is operated by the the Association of Universities for Research in Astronomy, Inc, under cooperative agreement with the National Science Foundation.

2 Stars $\mathrm{F}, \mathrm{U}, \mathrm{W}$, and $\mathrm{X}(B V I)$.

${ }^{3}$ Stars 5, 6, 8, 10, 12, 2109 and $16(U B V)$.
}

standard $U B V I$ photometric system are:

$$
\begin{aligned}
& V=v-0.004( \pm 0.033)(b-v)+0.005( \pm 0.023), n=17 \\
& V=v-0.039( \pm 0.021)(v-i)+0.036( \pm 0.031), n=8 \\
& U-B=1.042( \pm 0.018)(u-b)-0.021( \pm 0.018), n=17 \\
& B-V=0.918( \pm 0.010)(b-v)+0.072( \pm 0.016), n=17 \\
& V-I=0.969( \pm 0.021)(v-i)+0.035( \pm 0.020), n=8
\end{aligned}
$$

The standard errors of the slope coefficients and constants are given along with number, $n$, of standard stars used for the respective transformations.

In our preliminary list, we retained only those stars that had at least two measurements per night in each photometric band. We included in our final list only those stars for which at least one color-index was determined. A total of 12487 stars were measured mainly in $V$. Of them, 7567 stars have $U-B$ color, 8623 have $B-V$, and 12163 have $V-I$. For stars of the brighter sequences, i.e. with $V<18.0$, the r.m.s. errors are, on average, \pm 0.015 in $U, \pm 0.020$ in $B, \pm 0.013$ in $V$, and \pm 0.011 in $I$.

From 12487 stars measured by us in NGC 3201, we have 8501 in common with the study by Layden \& Sarajedini (2003). For a subsample of the brighter stars of this sample, with $V<$ 18.0, we obtained the following estimates of the mean differences between the two photometries, and the number of stars employed, those of this work minus that of Layden \& Sarajedini: $\delta V=+0.0030( \pm 0.0023), 2464 ; \delta(B-V)=+0.0150( \pm 0.0012)$, $2381 ; \delta(V-I)=+0.0080( \pm 0.0012), 2391$. The quoted uncertainties are standard errors of the mean. The overall agreement is good for virtually the entire range of the $V$ magnitude, although some systematic differences can only be noted for extreme colors. In particular, we see evident systematic differences between the two photometries in both the $(V-I)$ color-index and $I$ magnitude of the brighter red giants. It is seen in the bottom panels of Fig. 1 that compares the two photometries, where the differences in colors and magnitudes are in the order of our data minus those of Layden \& Sarajedini (2003). The stars used to determine the transformation from instrumental color and magnitudes to the standard $U B V I$ photometric system are in the range $10.9<V<$ 15.9, $0.0<U-B<1.5,0.4<B-V<1.4,0.6<V-I<1.4$. The differences are somewhat smaller, particularly for fainter stars, if we compare stars in regions with least crowding, at the radial distance out of 350 pix. of the cluster center. As noted, we used standard stars located in the observed field of NGC 3201. To check which of the two photometries is more likely responsible for the demonstrated disagreements, particularly in the $(V-I)$ color-index, we additionally compared our photometry with that by Stetson (2000) of around 300 cluster stars in 

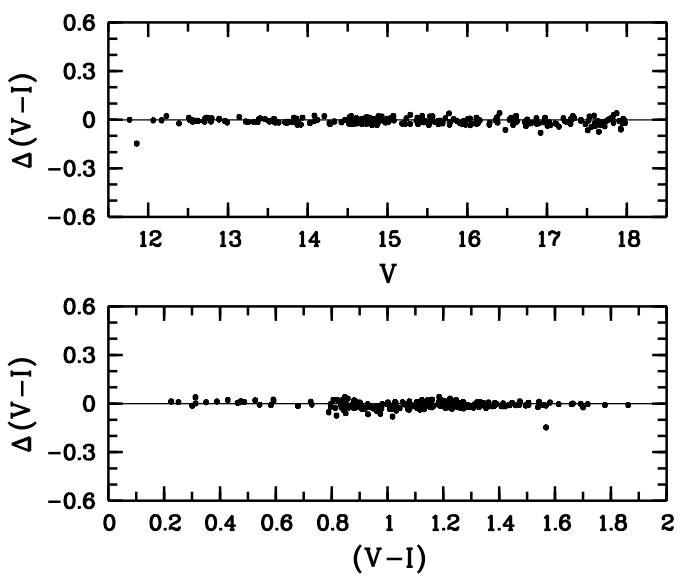

Fig. 2. Comparison of our present photometry of NGC 3201 and photometry by Stetson (2000) in the cluster, taken from Stetson Photometric Standard Fields. The difference in the color $V-I$ is in the order of our data minus those of Stetson.

$B V I$. It was taken from Stetson Photometric Standard Fields available at http: //www3. cadc-ccda.hia-iha.nrc-cnrc. gc.ca/community/STETSON/standards/. We were able to identify the bulk of the stars. This comparison for 257 stars with $V<18.0 \mathrm{mag}$ is shown in Fig. 2. It demonstrates very good agreement between the two photometries in the $(V-I)$ color, in its wide range, and there are no obvious effects like those seen in Fig. 1. The comparison also confirms really good accuracy of our photometry at least in the $V$ an $I$ bands above the $V$-magnitude of the turnoff point. Mean differences between the two photometries, in the sense: this work minus that of Stetson (2000), and the number of stars with $V<18.0$ utilized for the calculations are as follows: $\delta V=+0.0122( \pm 0.0022), 257 ; \delta(B-V)=$ $-0.0117( \pm 0.0018), 239 ; \delta(V-I)=-0.0092( \pm 0.0013), 257$. The uncertainties are standard errors of the mean.

\section{The color-magnitude diagrams and factors affecting them}

\subsection{The original color-magnitude diagrams}

The $V-(B-V), I-(V-I)$, and $U-(B-I)$ CMDs shown in three panels of Fig. 3 combine various pairs of magnitudes and colors obtained from our photometry of more than 12000 stars in the observed field of NGC 3201. The appearance of the principal sequences in the CMDs is affected by differential reddening, the presence of which was revealed and examined in previous studies mentioned in Sect. 1. In particular, the apparent width of the RGB is somewhat increased, as compared to a typical one in GCs unaffected by differential reddening. Moreover, it depends on the color index, because it is narrower in the $(B-V)$ color and wider in the $(B-I)$ color than in the $(V-I)$.

The CMDs are also contaminated by field stars. Their proportion naturally increases with increasing radial distance from the cluster center, since the projected density of the cluster stars decreases in the same direction. The changing appearance of the cluster's CMDs at different radial distance implies that the total number of the field stars contaminating the low RGB can be noticeable in a sample of the red giants populating the observed cluster field, especially the outer parts of NGC 3201. To minimize the negative effect of the two above-mentioned factors on the reliability of the results of our study, we first examined the effect and reduced it as much as possible.

\subsection{Correction of color-magnitude diagrams for differential reddening}

To examine reddening variations in the observed cluster field, we divided the field by ring shaped segments (RSSs) drawn around the center of NGC 3201. With this aim we first estimated the position of the cluster center and found it at $x=1700$ pixel and $y=880$ pixels. Then we divided the cluster field by (1) eight sectors, from S1 trough S8 and (2) a sequence of concentric rings of increasing radius with their center coinciding with the accepted center of the NGC 3201, the concentric circles being drawn at the radial distances of 0.68, 2!.05, 3'.42, and 5!47 (100, 300, 500, and 800 pixels, respectively) from the center. Therefore, the cluster field was finally composed of one central circular zone with a radius of $R=100$ pixels and sets of consecutive RSSs in each of the eight sectors. This composition of the observed field of ring-shaped segments is shown in Fig. 4.

Such a design was mainly chosen to partially compensate for the decrease in the projected surface density of stars towards the outer parts of the cluster by simultaneously increasing the area of each subsequent individual RSS in the same direction. With this, we aimed at reproducing, in the respective individual CMDs of these RSSs, not only the main sequence but also the brighter, less populated sequences, namely the sub-giant branch (SGB) and the RGB, at least its lower part. It is evident that the more reliably these sequences are traced in the individual CMDs of the RSSs, the more reliable is estimating shift in color and magnitude between the mean locus of the sequences in the CMDs.

We then compared the location of these sequences in all individual CMDs and evaluated shifts between them in different colors and magnitudes. With the purpose of the subsequent analysis of our photometry, the shifts were estimated in three colors, $(B-V),(V-I)$, and $(B-I)$, and two magnitudes, $V$ and $U$, by examining sets of six individual CMDs for each separate RSS. As a result, we conditionally defined four grades (levels) of reddening in the cluster field, in accordance with the estimated shifts, provided reddening is what is fully responsible for these shifts. We isolated regions with the same mean reddening. They are marked by four colors in the reddening map in Fig. 4. The regions shown by blue, green, yellow, and red symbols correspond to four grades of progressively increasing reddening. There are two RSSs belonging to the regions of the "green" mean reddening but with increased dispersion of stars in their CMDs. These RSSs are located in sectors S1 and S6. They are denoted by crosses in Fig. 4.

The original individual CMDs of the RSSs composing cluster regions of a given reddening were combined without any corrections applied. Therefore, we obtained four intermediate sets of CMDs for the regions characterized by four different grades of reddening, i.e. "blue", "green", "yellow", and "red". To make last step and to combine, in turn, these CMDs, we estimated offsets in colors and magnitudes between the "red" CMDs, on the one hand, and the "blue", "green", and "yellow" ones, on the other hand. Then, the latter CMDs were reduced to the "red" ones used as reference CMDs. We those obtained final set of the CMDs for the entire cluster field, corrected for differential reddening. These offsets are listed in Table 1. The most probable colors and magnitudes (as estimated from CMDs with different colors) of the turnoff (TO) point in the reference "red" CMDs are as follows: $V=18.35, U=19.05, B-V=0.70, V-I=0.94$, $B-I=1.64$. The typical internal accuracy of these estimates is approximately $0.007 \mathrm{mag}$ in color and 0.05 mag in magnitude. In turn, the offsets' uncertainties are on the order of 0.01 mag in 

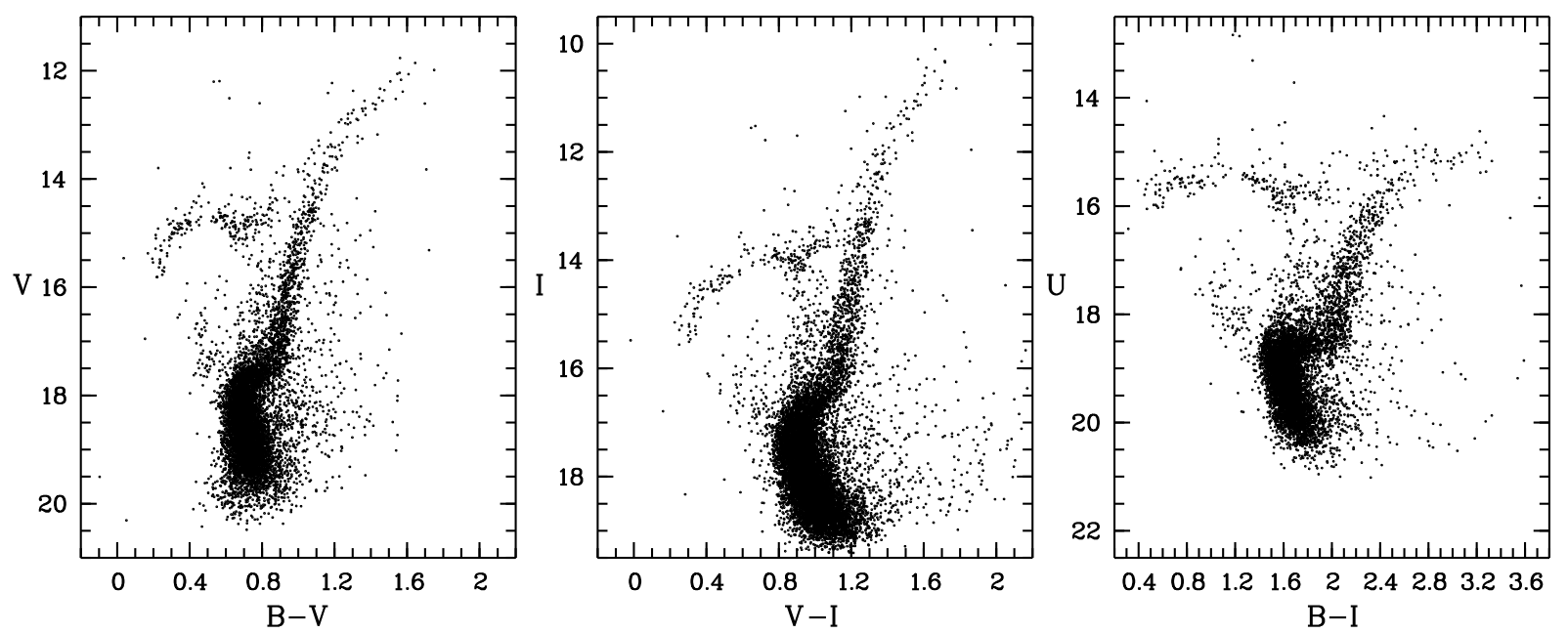

Fig. 3. The original $V-(B-V), I-(V-I)$ and $U-(B-I)$ CMDs based on $U B V I$ photometry of stars in a $14^{\prime} \times 14^{\prime}$ field of the GC NGC 3201 .

Table 1. Data on color and magnitude offsets applied to the CMDs of regions with different reddening.

\begin{tabular}{cccccc}
\hline \hline $\begin{array}{c}\text { Reddening } \\
\text { grade }\end{array}$ & $\begin{array}{c}\Delta(B-V) \\
\text { mag }\end{array}$ & $\begin{array}{c}\Delta(V-I) \\
\text { mag }\end{array}$ & $\begin{array}{c}\Delta(B-I) \\
\text { mag }\end{array}$ & $\begin{array}{c}\Delta V \\
\text { mag }\end{array}$ & $\begin{array}{c}\Delta U \\
\text { mag }\end{array}$ \\
\hline blue & 0.04 & 0.09 & 0.15 & 0.20 & 0.25 \\
green & 0.02 & 0.04 & 0.07 & 0.10 & 0.15 \\
yellow & 0.01 & 0.01 & 0.02 & 0.05 & 0.05 \\
\hline
\end{tabular}

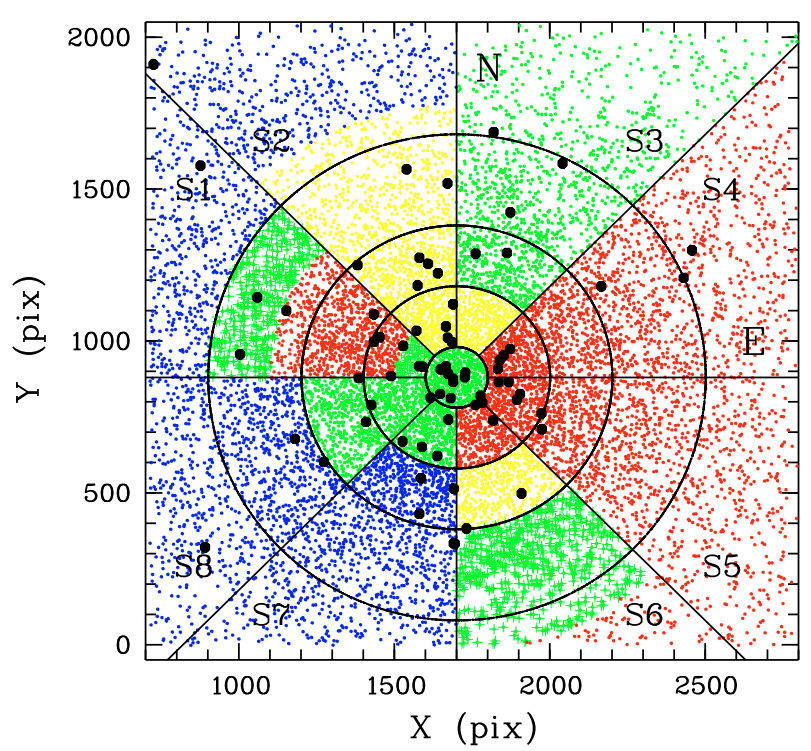

Fig. 4. The map of the reddening variations estimated in the observed field $\left(14^{\prime} \times 14^{\prime}\right)$ of the GC NGC 3201 and the positions of BS candidates (black filled circles) in the same cluster field. The regions shown by blue, green, yellow, and red symbols correspond to progressively increasing reddening. The observed cluster field is divided by eight sectors from S1 trough S8. The concentric circles are drawn at the radial distances of 0.68, 2'.05, 3.42, and 5'.47 (100, 300, 500, and 800 pixels, respectively) from the center of NGC 3201.

color and 0.07 in magnitude. These uncertainties do not account for possible systematic errors due to the effects of residual differential reddening within areas of the same grade of reddening, i.e. the same color in Fig. 4.

Some of the ratios $\Delta(\mathrm{mag}) / \Delta$ (color) calculated using data from Table 1 deviate from standard ones; however, this does not mean that the reddening law is nonstandard in the direction to the cluster. It is easy to see that offsets, which would correspond to a standard reddening law, are within or close to the errors of the offsets.

We note good overall agreement between our reddening map and the corresponding part of the map obtained by von Braun \& Mateo (2001) for a larger field around NGC 3201. In particular, we find very similar locations of the regions with the lowest and highest reddening, including a patch of high reddening located in sector S1. Some visible disagreements between the two maps seem to concern the variations of reddening with radius in the region corresponding to sector S6 in our map.

In the map by Braun \& Mateo (2001), we identified and isolated four samples of pixels that belong to the areas of the cluster field corresponding to four different grades of reddening in our map. The "red", "yellow", "green", and "blue" samples includ $27[(11-12) * 14+(10-13) * 15+(8-14) *(16-18)], 13$ $[(7-8) *(9-11)+9 *(10-11)+10 *(11-13)+14 *(13-14)]$, $25[5 *(13-18)+6 *(13-17)+7 *(13-16)+(8-9) *$ $(13-15)+12 *(9-11)+13 * 9]$, and $28[5 *(7-11)+(6-$ $13) * 7+(14-16) *(7-11)]$ pixels, respectively. First and second factors in each their pair given in square brackets indicate (a range of) the sequential number(s) of pixel(s) in the $x$ and $y$ axes, respectively, in the map by Braun \& Mateo (2001). We then calculated the averaged reddening for each of the four samples and the differences of reddening in the color $(V-I)$ (like those listed in Table 1) between the area with "red" grade of reddening, on the one hand, and the areas with "blue", "green", and "yellow" ones, on the other. They are $0.105( \pm 0.008), 0.064( \pm 0.007)$, and $0.024( \pm 0.009) \mathrm{mag}$, respectively, as compared to $0.09,0.04$, and 0.01 mag listed in the third column of Table 1, with their uncertainty $\sim 0.01 \mathrm{mag}$. The uncertainties quoted in parentheses are standard errors of the mean. Although the apparent slight disagreement between the two sets of reddening differences is close to the errors, some systematic effects cannot be ruled out. In particular, we cannot exclude somewhat more weight attributed to reddening in those RSSs that are localized closer to the cluster center, since they contain more (lower) red giants than in the outer cluster parts. We find that two of three reddening differences, namely "red"-"blue" and "red"-“yellow", become formally indistinguishable in the two maps for subsamples of pixels ( $\sim 10$ units) localized closer to the cluster center.

The resulting $V-(B-V), V-(V-I)$, and $U-(B-I)$ CMDs corrected for differential reddening in the observed field 

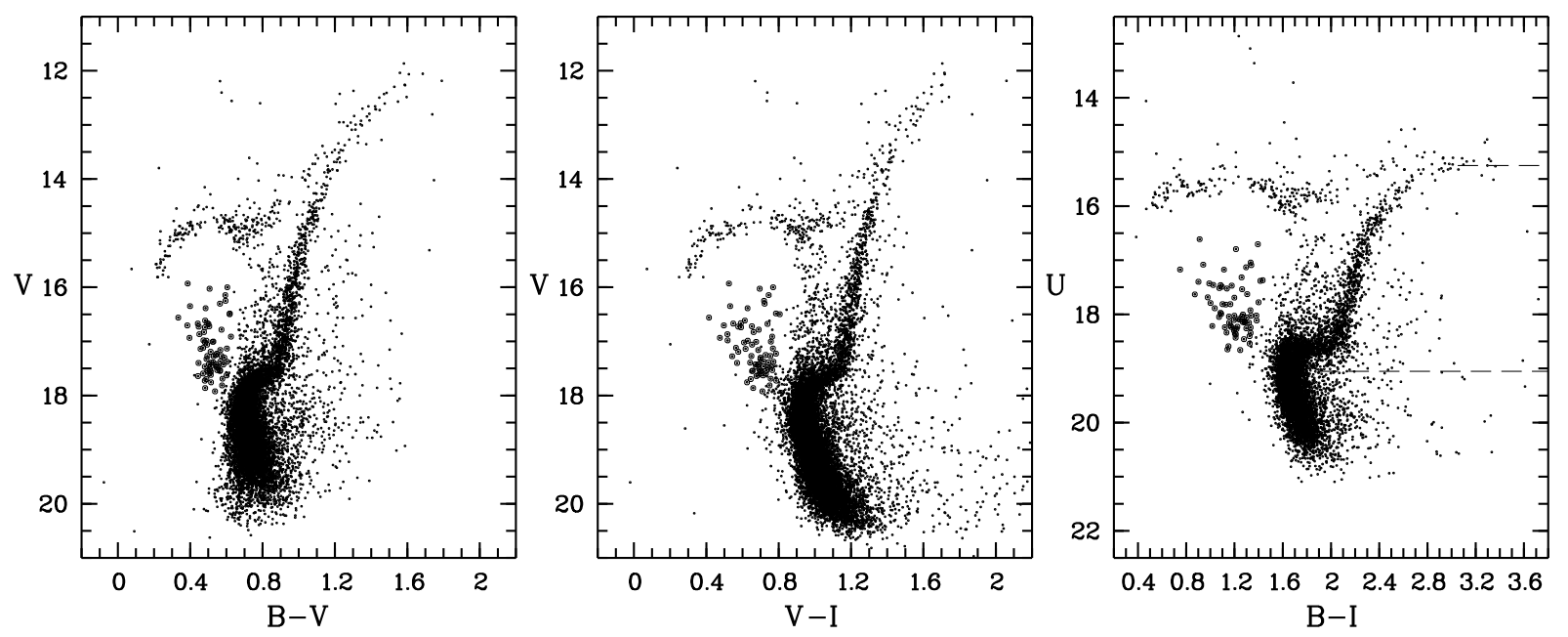

Fig. 5. The $V-(B-V), V-(V-I)$, and $U-(B-I)$ CMDs of NGC 3201, corrected for differential reddening in the observed cluster field, as described in detail in the text. Blue straggler candidates are shown by circles in the diagrams. In the right panel the $U$-levels of the RGB inflection point and the turnoff point are shown by the upper and lower horizontal dashed lines, respectively, aimed at demonstrating the metallicity-sensitive parameter $\Delta U_{\mathrm{TO}}^{\mathrm{RGB}}$.

of NGC 3201 are shown in the three panels of Fig. 5. The principal sequences are obviously narrower in these CMDs than in the original ones, particularly in the color $(B-I)$, in which the original CMD showed the largest width of the RGB. It should be noted, however, that the spread of stars around the RGB tip is conserved and does not disappear. Two very probable contributors (but not obviously main ones) to this spread can be (1) the residual effect of differential reddening and (2) variability of stars at the RGB tip, found by Layden \& Sarajedini (2003) for NGC 3201. However, the typical amplitude of their variability is a few hundredths of magnitude in the $V$ passband, so an additional contributor cannot be excluded, such as the possible spread in metallicity among stars suspected and discussed in earlier publications.

\subsection{Decontamination of field stars}

Beside the correction of CMDs for differential reddening in the field of NGC 3201, we were able to reduce their contamination by field stars. Figures 3 and 5 assume that the contamination can be non negligible in the lower RGB; therefore, it is capable of affecting the results of our study devoted to BSs (Sect. 4) and to the luminosity function (LF) of the RGB (Sect. 5). We made use advantages of multi-band photometry, particularly in the $U$ passband, for decontaminating the lower RGB from field stars. For this purpose, we applied the two-color diagram.

The $(U-B)-(B-I)$ two-color diagram in the left panel of Fig. 6 clearly shows that the sequence of RGB stars is separated from one another, more diffuse one formed by stars, the bulk of which are field stars. Both sequences are almost parallel to each other until the point, corresponding to the base of the RGB, where the bending occurs from the RGB to a horizontal sequence formed by the SGB stars. In the two-color diagram, we selected stars forming this "diffuse" sequence, by distinguishing them from RGB stars and from the majority of SGB stars. The selected stars are shown by red dots in Fig. 6. To check their location in CMD, we plotted these stars in the $V-(B-I)$ plane presented in right panel of the figure. It is seen that the majority of these stars can more or less obviously be distinguished from stars of the principal sequences, but some of them are indistinguishable from the lower RGB stars in the CMD.
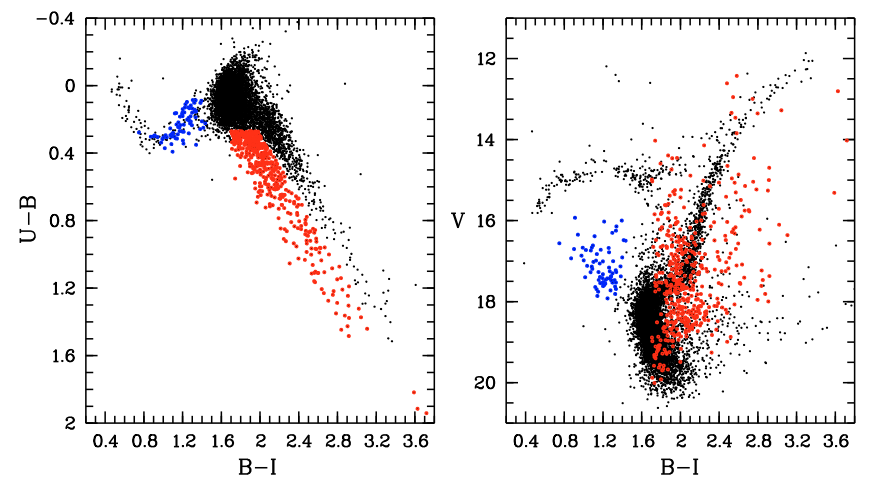

Fig. 6. Left panel: the $(U-B)-(B-I)$ two-color diagram for the observed field of NGC 3201. Red dots are stars, the majority of which are field stars forming a sequence parallel to that of the cluster's red and asymptotic giant stars; blue dots are the blue straggler candidates. Right panel: the $V-(B-I) C M D$ displaying the location of the same stars as in the two-color diagram. The $(B-I)$ color and $V$ magnitude are corrected for differential reddening in the cluster field.

We used two-color diagrams as a valuable decontaminating tool that helps reveal very probable field stars apparently coinciding with RGB stars in CMDs but deviating significantly from this sequence towards the "diffuse" one in the two-color plots.

\section{Blue stragglers}

Relying on four-color photometry of the cluster we was able to isolate blue straggler (BS) candidates more confidently than in the case of two-color photometry. This was achieved thanks to the requirement of the simultaneous location of the candidates within the region of the most probable concentration of BSs in several CMDs with different color-indices, namely $(B-V)$, $(V-I)$, and $(B-I)$. Doing so, we isolated 73 probable candidates. It is the most numerous population of these stars found in NGC 3201, considering that Brewer et al. (1993) found 31 BS candidates. Their position in the cluster field is marked in Fig. 4, while their location in the CMDs corrected for differential reddening is shown in Fig. 5. 


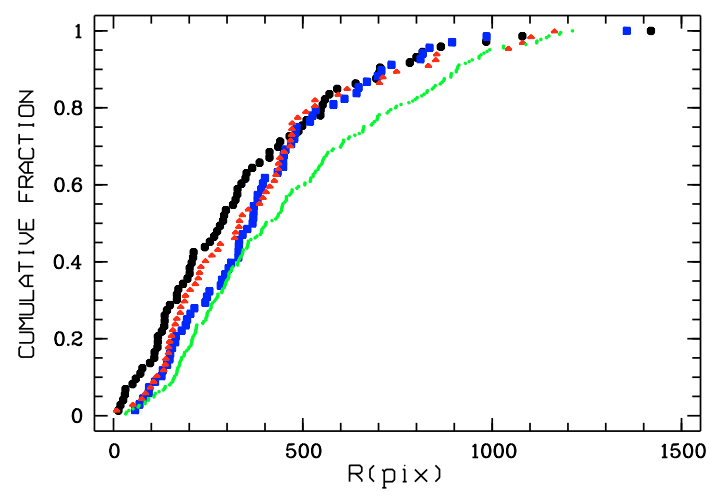

Fig. 7. A comparison of the cumulative radial distributions of blue straggler candidates (black filled circles), blue HB stars (blue filled squires), RGB stars in the luminosity range of the RGB bump (red filled triangles), and lower RGB stars (green dots) with brightness comparable to, but somewhat higher than that of the blue straggler candidates.

We compared the cumulative radial distribution of the isolated BS candidate with those of three samples of stars belonging (1) to the lower RGB (225 stars in the magnitude range $16.0<V<17.0)$, (2) to the brighter RGB, in the region of its bump (67 stars in the magnitude range $14.4<V<15.1$ ), and (3) to the BHB (68 star in the magnitude range $14.7<V<15.9$ ). The BS candidates fall in the magnitude range $15.8<V<18.0$. The two samples of the RGB stars were taken from the total sample of RGB stars obtained to construct RGB LF, as described in detail in Sect. 5. These samples were decontaminated from field stars using the two-color diagram, as described above, in Sect. 3.3. Figure 7 shows the cumulative radial distributions of BS candidates and of the mentioned stars. One can see that the BS candidate are obviously more centrally concentrated than the lower RGB stars. This apparent difference between both distributions is confirmed by Kolmogorov-Smirnov test: the difference is statistically significant at a $99.2 \%$ confidence level. This estimate is instead the lower limit, since the lower RGB stars are, on average, brighter than the BS candidates. It is interesting to note that the difference between both distribution is greater if the decontamination of the RGB stars from field stars is not applied. Despite a significant difference in mean brightness (exceeding 2 mag in the $V$ ) between the population of $\mathrm{BS}$ candidate, on the one hand, and the populations of both the BHB and upper RGB stars, on the other, some difference between the cumulative radial distributions of the former population and the latter ones (in particular, of BHB star population) is conserved.

The BS candidates appear in the magnitude range of about 2 mag. in both $U$ and $V$ in the CMDs corrected for differential reddening. The majority of them are localized closer to the fainter end of the range, in a fairly narrow region, by forming an apparent clump. It is located around $\sim 0.8-0.9$ mag brighter than the estimated magnitudes of the TO point in the $U$ and $V$ passbands. At the same time, the candidates do not show any notable segregation between them in the two-color diagram, as can be seen in Fig. 6. In other words, in contrast to some more concentrated GCs, BS in NGC 3201 do not demonstrate any obvious separation in the two-color diagram, which might imply the presence of their groups with a different BSs formation mechanism. We found two such groups in M80 (Alcaíno et al. 1998) by following the approach of Lauzeral et al. (1993), who in turn had applied the method of Bailyn (1992) to isolate BSs of probable collisional origin. We conclude that among the BS candidate

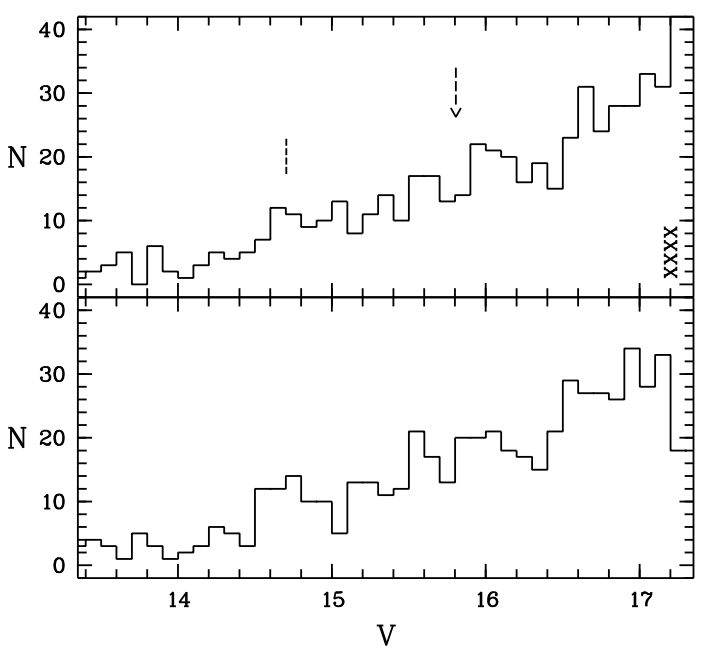

Fig. 8. A comparison of the RGB LF of NGC 3201 in the $V$ passband, corrected (the upper panel) and uncorrected (the lower panel) for differential reddening. The arrow shows the universal dip in the lower RGB LFs of GCs, while the short-dashed line indicates the position of the RGB bump; " $x$ "-sign composed column marks the position of the RGB base.

isolated by us in large field of NGC 3201, there is none with a corresponding position in the two-color diagram (i.e., with bluer $U-B$ color), which would suggest the collisional nature of its origin.

The isolated BS candidate are marked by flags in the table with the photometric data, which is available in electronic form. We were able to identify 21 stars in common with the BS candidates found by Brewer et al. (1993). In the same table we list the sequential numbers that the identified stars have in Table 3 of Brewer et al. (1993).

\section{The red giant branch luminosity function}

To construct and analyze the RGB LF of NGC 3201, we have tried to avoid the contamination of the RGB by (1) stars belonging to both the asymptotic giant branch and red HB, (2) field stars that appear among the RGB stars on CMD with a given color-index, but are displaced from the sequence in CMDs with other color-indices or/and on the two-color diagrams, as shown in Sect. 3.3, or (3) stars showing large deviation from the sequence's fiducial line due to photometric error. With this aim, we used the advantage of a multi-color photometry and proceeded in the following way. In the $V-(B-I) \mathrm{CMD}$, we fitted the mean locus of the RGB with a polynomial applying corresponding commands in the MIDAS system. We next linearized the RGB by subtracting for each star the color of the mean locus at its luminosity level from the star's color-index. We left only those stars that satisfied our selection criterium: their deviations, $\delta(B-I)$, from the mean locus did not exceed \pm 0.075 mag at and above the level of the HB, and \pm 0.10 mag. below it. These conditional boundaries of the RGB separates the bulk of its stars from the majority of stars belonging to the asymptotic and red horizontal branches and are close to the mean errors in the colors along the RGB. It cannot be excluded that some stars really belonging to the RGB near its tip were rejected due to the above-mentioned increased dispersion of them. Finally, we made second step of decontamination applying the two-color diagram (and CMDs with different color-indices) and rejecting deviating stars. 
In the upper and lower panels of Fig. 8 we show two versions of the RGB LF of NGC 3201 in the $V$ passband, namely corrected and uncorrected for differential reddening, respectively. First of all, we note the appearance of the well known RGB bump normally causing a more or less obvious peak in the RGB LFs of GCs. It was the first feature in RGB predicted and explained in the frames of theory of stellar evolution (for more detail and for references see, for example, Sweigart \& Gross 1978). It is seen that the bump in the uncorrected LF is more broadened and its position somewhat less definitive than in the corrected LF. Note the fairly clear appearance of the clump of RGB stars, corresponding to the bump, in the intermediate panel of Fig. 5. Despite its better shaping and localization in the latter LF, the bump still seems to be less pronounced than typically in RGB LFs constructed using the same bin and of comparable sample size in GCs with similar metallicity. A residual differential reddening affects the bump's appearance. Meanwhile, taking a possible metallicity spread in NGC 3201 into account, we do not exclude the cluster's intrinsically broadened, hence additionally smoothed, RGB bump.

In Fig. 8 we also direct attention to an apparent dip located at $\Delta V_{\text {base }}^{\text {dip }}=1.4$ mag above the RGB base. A dip with the same, on average, location relative to the RGB base and quite weak dependence on metallicity has been recently detected by Kravtsov (2008) using a sample of the RGB LFs of GCs with the available homogeneous HST photometry. The dip shows up at the highest confidential level in the generalized RGB LF of metalpoor GCs with blue horizontal branch morphology. At the same time, it tends to be least pronounced in the generalized LF of GCs with intermediate HB morphology like that of NGC 3201. It is indeed fairly weak in the obtained LF of NGC 3201 and, strictly speaking, is statistically insignificant according our estimate. The RGB bump has marginal statistical significance.

\section{Metallicity}

In Kravtsov et al. (2007), we defined parameter $\Delta U_{\mathrm{TO}}^{\mathrm{RGB}}$ as the difference in the $U$ magnitude between the TO point and the inflection point of the RGB in the $U$-based CMDs of GC in metalpoor range, at $[\mathrm{Fe} / \mathrm{H}]_{Z W}<-1.1 \mathrm{dex}$ in the scale of Zinn \& West (1984), the color difference $\Delta(B-V)$ between these points being around $\Delta(B-V) \approx 0.8$ and almost independent of metallicity in the given range. We found that the parameter $\Delta U_{\mathrm{TO}}^{\mathrm{RGB}}$ does not obviously depend on the characteristics of $U$ filters used, at least within insignificant reddening and provided that the filters do not have a significant red leak, but it very tightly correlates with GC metallicities. We also calibrated $\Delta U_{\mathrm{TO}}^{\mathrm{RGB}}$ on metallicity and deduced an analytical relation between $\Delta U_{\mathrm{TO}}^{\mathrm{RGB}}$ and $[\mathrm{Fe} / \mathrm{H}]_{Z W}$.

Here we apply this new approach to estimate the metallicity of NGC 3201. To determine the parameter $\Delta U_{\mathrm{TO}}^{\mathrm{RGB}}$, we relied on the cluster $U-(B-V)$ CMD corrected for differential reddening. However, to estimate the $U$ magnitude of the turnoff point as carefully as possible, we examined its position in CMDs with different color indices. We find the $U$-levels of the RGB inflection point and of the turnoff point at $U_{\mathrm{RGB}}=15.25 \pm 0.10$ and $U_{\mathrm{TO}}=19.05 \pm 0.13$, respectively, as shown in the $U-(B-I)$ diagram presented in the right panel of Fig. 5. Hence the parameter is close to $\Delta U_{\mathrm{TO}}^{\mathrm{RGB}}=3.80 \pm 0.16 \mathrm{mag}$. Substituting this value to the analytical relation between $\Delta U_{\mathrm{TO}}^{\mathrm{RGB}}$ and metallicity, we obtain $[\mathrm{Fe} / \mathrm{H}]_{Z W}=-1.54 \pm 0.12$ dex. This value falls between extreme estimates of the cluster's metallicity obtained using different methods or indicators, since slightly more metal-poor than median value, as can be concluded from recent publications containing overviews of metallicity estimates in NGC 3201 (e.g., Piersimoni et al. 2002). The present estimate of the cluster's metallicity is in very good agreement with the $\left([\mathrm{Fe} / \mathrm{H}]_{Z W}=-1.55\right)$ made by us earlier (Samus et al. 1996), exploiting the dependence of the RGB bump position on metallicity. Also, it is very close to $[\mathrm{Fe} / \mathrm{H}]=-1.56$ derived by $\mathrm{Kraft}$ \& Ivans (2003) by reevaluating cluster metallicity based on high dispersion spectroscopy, and is in fair agreement (but within the errors) with $[\mathrm{Fe} / \mathrm{H}]=-1.36 \pm 0.14$ spectroscopically determined by Covey et al. (2003).

Our estimate of metallicity of NGC 3201 coincides with the one by Layden \& Sarajedini (2003) using their $V-(B-V)$ CMD of the cluster. At the same time, they obtained a notably lower value of cluster metallicity, differing by $0.35 \mathrm{dex}$, relying on CMD with the $(V-I)$ color. We find a very probable natural explanation of this contradiction by analyzing the comparison of our photometry, on the one hand, with those by Layden \& Sarajedini (2003) and by Stetson (2000), on the other hand, shown in Figs. 1 and 2, respectively, and discussed in Sect. 2. The comparison in Fig. 1 reveals a systematically increasing difference between the two photometries in the color $(V-I)$ with decreasing magnitudes of the upper red giants, in the sense that the $(V-I)$ color in the photometry by Layden \& Sarajedini (2003) gets bluer with decreasing magnitude, as compared to the giants' color in our photometry. Hence the upper RGB becomes artificially somewhat steeper in their CMD with the $(V-I)$ color, since it is their photometry that is very likely to be mainly responsible for the discussed trend in the $(V-I)$, considering the very good agreement of our photometry with that by Stetson (2000).

We likewise estimated metallicity of the cluster by exploiting the dependence of the position of the RGB bump on metallicity. This dependence was empirically obtained for the first time by Kravtsov (1989). Here, we apply the analytically expressed relation between metallicity and $\Delta V_{\text {bump }}^{\mathrm{ZAHB}}$, the magnitude difference between the bump, and the level of the zero-age HB, taken from Ferraro et al. (1999). In the CMD corrected for differential reddening, we estimate the locations of the bump (see Fig. 8) and ZAHB to be at $V_{\text {bump }}=14.70 \pm 0.07$ and $V_{\text {ZAHB }}=14.85 \pm 0.07$, respectively. Hence $\Delta V_{\text {bump }}^{Z A H B}=-0.15 \pm 0.10$ mag., which is converted to $[\mathrm{Fe} / \mathrm{H}]=-1.46 \pm 0.15 \mathrm{dex}$. It agrees with the metallicity value deduced from the parameter $\Delta U_{\mathrm{TO}}^{\mathrm{RGB}}$.

\section{Conclusions}

We obtained new $U B V I$ photometry of more than 12000 stars in a wide field, about $14^{\prime} \times 14^{\prime}$, of the southern GC NGC 3201 , below its turnoff point in all passbands. We exploited this multicolor photometry and its advantages over two-color photometry to further study this nearby GC known to have irregularly varying reddening in its field. We examined reddening variations in the observed cluster field, as well as the location of field stars in the $U$-based two-color diagrams and in CMDs with different color-indices. We were able to reduce the negative impact of both the irregularly varying reddening and contamination of the lower RGB by field stars. This permitted us to achieve the following main results, among others, and make the following conclusions.

We estimated metallicity of NGC 3201 using a new metallicity indicator recently proposed by us. It is related to deep, $U$ based CMDs of GCs that simultaneously reach the TO point and trace well the upper RGB with sufficient amount of its stars. We obtain $[\mathrm{Fe} / \mathrm{H}]_{Z W}=-1.54 \pm 0.12$ dex. We also find 
$[\mathrm{Fe} / \mathrm{H}]_{Z W}=-1.46 \pm 0.15$, by exploiting the dependence of the position of the RGB bump on metallicity. These estimates agree very closely with each other and with other estimates of the cluster's metallicity using different methods or indicators on the same metallicity scale. They are agree (within the errors) with spectroscopically determined cluster metallicity. We also find very probable natural explanation of the notable disagreement between metallicity estimates made by Layden \& Sarajedini (2003) using their CMDs with the $(B-V)$ and $(V-I)$ color-indices, respectively.

We isolated $73 \mathrm{BS}$ candidates, the largest in their population found in the cluster so far. They are found to be more centrally concentrated than the lower red giants. This difference, according to the Kolmogorov-Smirnov test, is statistically significant at the $99.2 \%$ confidence level. Their position in the $U$-based twocolor diagram shows that among them there are none that could belong to BSs with the collisional mechanism of their formation.

We briefly discuss the RGB LFs and two of its features, the RGB bump and a dip located above the RGB base at $\Delta V_{\text {base }}^{\text {dip }}=$ $1.4 \mathrm{mag}$.

Acknowledgements. FA is very grateful to Prof. F. Mirabel, representative of ESO in Chile, for kindly providing computing facilities at the ESO office in Santiago, where the observations were reduced. We thank the anonymous referee for useful comments that improved the manuscript. This research used the facilities of the Canadian Astronomy Data Centre operated by the National Research Council of Canada with the support of the Canadian Space Agency.

\section{References}

Alcaíno, G., \& Liller, W. 1984, AJ, 89, 1712

Alcaíno, G., Liller, W., \& Alvarado, F. 1989, A\&A, 216, 68

Alcaíno, G., Liller, W., Alvarado, F., et al. 1998, AJ, 116, 2415

Bailyn, C. D. 1992, ApJ, 392, 519

Brewer, J. P., Fahlman, G. G., Richer, H. B., Searle, L., \& Thompson, I. 1993, AJ, 105, 2158

Covey, K. R., Wallerstein, G., Gonzalez, G., Vanture, A. D., \& Suntzeff, N. B. 2003, PASP, 115, 819

Ferraro, F. R., Messineo, M., Fusi Pecci, F., et al. 1999, AJ, 118, 1738

Harris, W. E. 1996, AJ, 112, 1487

Kraft, R. P., \& Ivans, I. I. 2003, PASP, 115, 143

Kravtsov, V. V. 1989, Soviet. Astron. Lett., 15, 356

Kravtsov, V. V. 2008, AJ, submitted

Kravtsov, V., Alcaíno, G., Marconi, G., \& Alvarado, F. 2007, A\&A, 469, 529

Layden, A. C., \& Sarajedini, A. 2003, AJ, 125, 208

Lauzeral, C., Auriere, M., \& Coupinot, C. 1993, A\&A, 274, 214

Lee, S. W. 1977, A\&AS, 28, 409

Mazur, B., Krzemiński, W., \& Thompson, I. B. 2003, MNRAS, 340, 1205

Piersimoni, A. M., Bono, G., \& Ripepi, V. 2002, AJ, 124, 1528

Samus, N. N., Kravtsov, V. V., Pavlov, M. V., et al. 1996, Astron. Lett., 22, 239

Schlegel, D. J., Finkbeiner, D. P., \& Davis, M. 1998, ApJ, 500, 525

Stetson, P.B. 1987, PASP, 99, 191

Stetson, P.B. 1991, DAOPHOT II Users Manual

Stetson, P.B. 2000, PASP, 112, 925

Sweigart, A. V., \& Gross P. G. 1978, ApJS, 36, 405

von Braun, K., \& Mateo, M. 2001, AJ, 121, 1522

von Braun, K., \& Mateo, M. 2002, AJ, 123, 279

Zinn, R., \& West M. J. 1984, ApJS, 55, 45 